\title{
Note
}

\section{Submicroscopic deletion involving the fibroblast growth factor receptor 1 gene in a patient with combined pituitary hormone deficiency}

\author{
Maki Fukami ${ }^{1)^{*}}$, Manami Iso ${ }^{1)^{*}}$, Naoko Sato ${ }^{1)}$, Maki Igarashi ${ }^{1)}$, Misuzu Seo ${ }^{2)}$, Itsuro Kazukawa ${ }^{3)}$, \\ Eiichi Kinoshita $^{4)}$, Sumito Dateki ${ }^{1), 4)}$ and Tsutomu Ogata ${ }^{1), 5)}$ \\ 1) Department of Molecular Endocrinology, National Research Institute for Child Health and Development, Tokyo 157-8535, \\ Japan \\ 2) Department of Molecular Bioscience, Faculty of Life Sciences, Kyoto Sangyo University, Kyoto 603-8555, Japan \\ 3) Department of Endocrinology, Chiba Children's Hospital, Chiba 266-0007, Japan \\ 4) Department of Pediatrics, Nagasaki University Graduate School of Biomedical Sciences, Nagasaki 852-8501, Japan \\ 5) Department of Pediatrics, Hamamatsu University School of Medicine, Hamamatsu 431-3192, Japan
}

\begin{abstract}
Combined pituitary hormone deficiency (CPHD), isolated hypogonadotropic hypogonadism (IHH), Kallmann syndrome (KS), and septo-optic dysplasia (SOD) are genetically related conditions caused by abnormal development of the anterior midline in the forebrain. Although mutations in the fibroblast growth factor receptor 1 (FGFR1) gene have been implicated in the development of IHH, KS, and SOD, the relevance of FGFR1 abnormalities to CPHD remains to be elucidated. Here, we report a Japanese female patient with CPHD and FGFR1 haploinsufficiency. The patient was identified through copy-number analyses and direct sequencing of FGFR1 performed for 69 patients with CPHD. The patient presented with a combined deficiency of GH, LH and FSH, and multiple neurological abnormalities. In addition, normal TSH values along with a low free T4 level indicated the presence of central hypothyroidism. Molecular analyses identified a heterozygous $\sim 8.5 \mathrm{Mb}$ deletion involving 56 genes and pseudogenes. None of these genes except FGFR1 have been associated with brain development. No FGFR1 abnormalities were identified in the remaining 68 patients, although two patients carried nucleotide substitutions (p.V102I and p.S107L) that were assessed as benign polymorphism by in vitro functional assays. These results indicate a possible role of FGFR1 in anterior pituitary function and the rarity of FGFR1 abnormalities in patients with CPHD.
\end{abstract}

Key words: Anterior pituitary, Gonadotropin, Gene deletion, Copy-number, Mutation

FIBROBLAST GROWTH FACTOR (FGF) receptor 1 (FGFR1) and its major ligand FGF8 are key molecules for the development of GnRH neurons and the olfactory system [1, 2]. Upon binding of FGF ligands, FGFR1 dimerizes and activates the Ras-MAPK and phospholipase $\mathrm{C} \gamma$ pathways, thereby controlling cell proliferation and differentiation [1, 2]. Genetic defects in FGFR1 and FGF8 have been shown to underlie isolated hypogonadotropic hypogonadism (IHH)

Submitted Jan. 15, 2013; Accepted Apr. 9, 2013 as EJ13-0023 Released online in J-STAGE as advance publication May 9, 2013

Correspondence to: Maki Fukami, M.D., Department of Molecular Endocrinology, National Research Institute for Child Health and Development, 2-10-1 Ohkura, Setagaya, Tokyo 157-8535, Japan. E-mail: fukami-m@ncchd.go.jp

* These authors are contributed equally to this work. and Kallmann syndrome (KS) [3-5]. To date, several FGFR1 abnormalities have been identified in patients with IHH/KS mostly as monoallelic mutations, although in a certain percentage of patients, they appeared as digenic or biallelic mutations [5].

It has recently been postulated that IHH, KS, septo-optic dysplasia (SOD), and combined pituitary hormone deficiency (CPHD) are genetically related conditions caused by abnormal development of the anterior

Abbreviations: CGH, comparative genomic hybridization; CPHD, combined pituitary hormone deficiency; FGF, fibroblast growth factor; FGFR, FGF receptor; FISH, fluorescence in situ hybridization; IHH, isolated hypogonadotropic hypogonadism; KS, Kallmann syndrome; MLPA, multiplex ligation-dependent probe amplification; SOD, septo-optic dysplasia; WT, wildtype 
midline in the forebrain $[6,7]$. Mutations in FGF8, PROKR2, and KAL1 have been identified in patients with IHH, KS, SOD, and/or CPHD [6, 7]. Furthermore, FGFR1 mutations have been detected in a few patients with SOD [7].

Until now, however, FGFR1 mutations have not been described in patients with CPHD, with the exception of a cytogenetically detectable large deletion involving FGFR1 that was identified in a single case [8]. Therefore, although a positive role of FGF-FGFR signaling in the proliferation of pituitary cells has been indicated by in vitro studies $[9,10]$, it remains to be determined whether genetic defects in FGFR1 lead to CPHD. To clarify this, we performed copy-number and mutation analyses of FGFR1 for patients with CPHD.

\section{Patients and Methods}

\section{Patients}

This study was approved by the Institutional Review Board Committee at the National Center for Child Health and Development and was performed after obtaining written informed consent. We studied 69 Japanese patients ( 37 males and 32 females aged 1-43 years) with combined deficiencies of at least two pituitary hormones and a normal karyotype. Two of the 69 patients had a family history of pituitary dysfunction. The 69 patients invariably manifested GH deficiency. In addition, most patients had deficiencies of TSH and/or prolactin. Apparent ACTH and ADH deficiencies were observed in 24 and 6 patients, respectively. Of 41 patients examined after the pubertal age of normal children, 32 showed compromised FSH/LH secretion. Brain magnetic resonance imaging delineated pituitary hypoplasia in 34 patients, and stalk interruption in seven. Eleven patients, including four with SOD, exhibited eye abnormalities. Seven patients manifested clinically discernible developmental delay. Patients with a history of birth trauma and those carrying protein-altering mutations or copy-number aberrations of POU1F1, PROP1, HESX1, LHX3, LHX4, OTX2, and SOX3 were excluded from this study. DNA samples of 100 healthy Japanese adults were utilized as controls.

\section{Copy-number analyses}

Genomic DNA samples were obtained from leukocytes of the patients. Multiplex ligation-dependent probe amplification (MLPA) was carried out using a commercially available MLPA probe mix (P133 kit; MRC-Holland, Amsterdam, The Netherlands). To confirm a deletion, we performed fluorescence in situ hybridization (FISH) analysis on lymphocyte metaphase spreads using a PCR-generated probe. The extent of a deletion was analyzed by an oligoarray-based comparative genomic hybridization $(\mathrm{CGH})$ using $1 \times 244 \mathrm{~K}$ human catalog array (G4411B; Agilent Technologies, Palo Alto, CA, USA). The size and location of a deletion were analyzed using the UCSC genome browser (http://genome.ucsc.edu/).

\section{Mutation analysis}

Direct sequence analysis was carried out for the coding exons and their flanking splice sites of FGFR1. The primers utilized in this study are shown in Table 1 . To confirm a heterozygous nucleotide substitution, the corresponding PCR products were subcloned using a TOPO TACloning Kit (Life Technologies, Carlsbad, CA, USA), and the two alleles were sequenced separately.

\section{Functional analysis of missense variants}

For missense variants, in vitro reporter assays were carried out by a previously reported method with modifications $[11,12]$. In brief, an expression vector for the wildtype (WT) FGFR1 was constructed by inserting the PCR-amplified cDNA of human FGFR1 into a pcDNA3. $1^{+}$vector (Life Technologies). An expression vector for the FGFR1 variants was generated by site-directed mutagenesis. A reporter vector was constructed by inserting the rat osteocalcin promoter sequence (from -206 to +38) into the PGL3-basic luciferase vector (Promega Madison, WI, USA). Transfection assays were carried out using rat L6 myoblast cells (CRL-1458, ATCC) that lacked endogenous Fgfr1 expression. The cells were maintained in DMEM containing $10 \%$ fetal bovine serum and seeded into 24 -well dishes. Transient transfection was performed using FuGENE 6 Reagent (Roche Diagnostics, Basel, Switzerland) with: (1) empty pcDNA3.1 $1^{+}$expression vector (200 ng), (2) WT FGFR1 expression vector (200 ng), (3) variant FGFR1 expression vector (200 ng), or (4) variant (100 ng) plus WT (100 ng) FGFR1 expression vectors, together with the luciferase reporter plasmids (60 ng) and a pRL-null vector ( $25 \mathrm{ng}$, Promega) used as an internal control. At 24 hours after transfection, the medium was replaced by fresh medium supplemented with 2.0 nM FGF8 (Wako, Osaka, Japan), $1.0 \mu \mathrm{g} / \mathrm{mL}$ heparin (Sigma-Aldrich, St. Louis, MO, USA), and $0.1 \%$ fetal bovine serum. The 
Table 1 Primers utelized in the present study

\begin{tabular}{|c|c|c|}
\hline & Forward primer & Reverse primer \\
\hline \multicolumn{3}{|l|}{ FGFR1 sequence analysis } \\
\hline Exon 2 & ССTTCTGTTTTTCСТTTCССC & GAGCCTTCCCTGTTGACCA \\
\hline Exon 3 & TCTTCСТCTCTCGCСССТT & AAGCAGAGTGGGGGCAGAT \\
\hline Exon $4+5$ & AAATCTAGGGGTCCCTAGGAG & TCСССТGTTCССАТТАСТСТА \\
\hline Exon 6 & GTCATGGGGCCTGCATTTT & AAGTGCCAATCGCTATCCTGA \\
\hline Exon 7 & ATGGCAAGGTCCCCATGA & AAGCGTGAGGAATGATCCCAT \\
\hline Exon 8 & AGGTCTCATGTCCTGTGCTTG & AGGATTCAGCCCTCAAAGCT \\
\hline Exon 9 & CAGGAGACAGGTGTTGCTTTT & AGCCAGAAAATAAGGCCCAA \\
\hline Exon $10+11$ & TTGGGAAGCCCTGACTAAGAA & TGTTTGCTTGGAATGGGACA \\
\hline Exon 12 & AAAGCAGCCCCTCGACACATA & ACCCCAGCTCAGATCTTCTCC \\
\hline Exon 13 & AACCTGCTCACCTGCTGCT & CACAGGCTGGAAGACTAGGG \\
\hline Exon 14 & CAGAGCAGTGTGGCAGAAGTT & ATGAGGGCACAGGTGGGAA \\
\hline Exon 15 & TGAGAACCCACCCCCCAGT & GCATGGAGAACAGATCAGCCT \\
\hline Exon 16 & TGGGTAGAGGATTTGTGCTGG & TCAGGGACCGTCTCCTGGAGAT \\
\hline Exon 17 & TAAGCCCGAGGAGATGTCG & AAAGCTGGGGGAGTACTGGT \\
\hline Exon 18 & CCATCGCTGCTTCCTCTC & TTACAGCTGACGGTGGAGTCT \\
\hline \multicolumn{3}{|l|}{ FISH probe } \\
\hline LA-PCR FGFR1 & AGTAAACAATAGGGTGAGAACTTTTGCT & CCATGTTATAGCATAGAATCAGCACTTT \\
\hline \multicolumn{3}{|l|}{ Reporter vector constuction } \\
\hline Osteocalcin promoter & ACGCGTAGGTCTGGGGCCAT & AGATCTGGTCTGCACCGAGT \\
\hline \multicolumn{3}{|l|}{ Mutagenesis } \\
\hline V102I & TATGCTTGCATAACCAGCAGCCССТC & GGTTATGCAAGCATAGAGGCCGGAGT \\
\hline S107L & CCCTTGGGCAGTGACACCACCTACTT & GTCACTGCCCAAGGGGCTGCTGGTTA \\
\hline
\end{tabular}

cells were cultured for an additional 18 hours and then subjected to luciferase analysis using the luciferase reporter assay system (Promega). Relative luciferase activity was measured with Lumat LB9507 (Berthold, Oak Ridge, TN, USA). These experiments were carried out in triplicate within a single experiment and the experiment was repeated three times. The results are expressed as the mean $\pm \mathrm{SEM}$, and statistical significance was determined by the $t$-tests. Values of $p \leq 0.05$ were considered significant.

\section{Results}

\section{Identification of a submicroscopic deletion involving FGFR1}

A heterozygous deletion involving FGFR1 was identified in one female patient. This deletion was indicated by MLPA and confirmed by FISH (Fig. 1A).
CGH revealed that the deletion was $\sim 8.5 \mathrm{Mb}$ in size and included all exons of FGFR1 (Fig. 1B). In addition to FGFR1, this deletion affected 55 genes and pseudogenes (Table 2). Parental samples of the patient were not available for genetic analysis. This deletion was not detected in the control group.

\section{Identification of two FGFR1 missense variants}

Direct sequence analysis identified two heterozygous variations, p.V102I (c.304 G>A) and p.S107L (c.320 $\mathrm{C}>\mathrm{T}$ ), in two female patients (Fig. 2A). The father of the p.V102I-positive patient also carried this variation, while parental samples of the p.S107L-positive patient were not available for genetic analysis. The p.V102I and p.S107L variants were found in two and one of the 100 control individuals, respectively. 


\section{A}
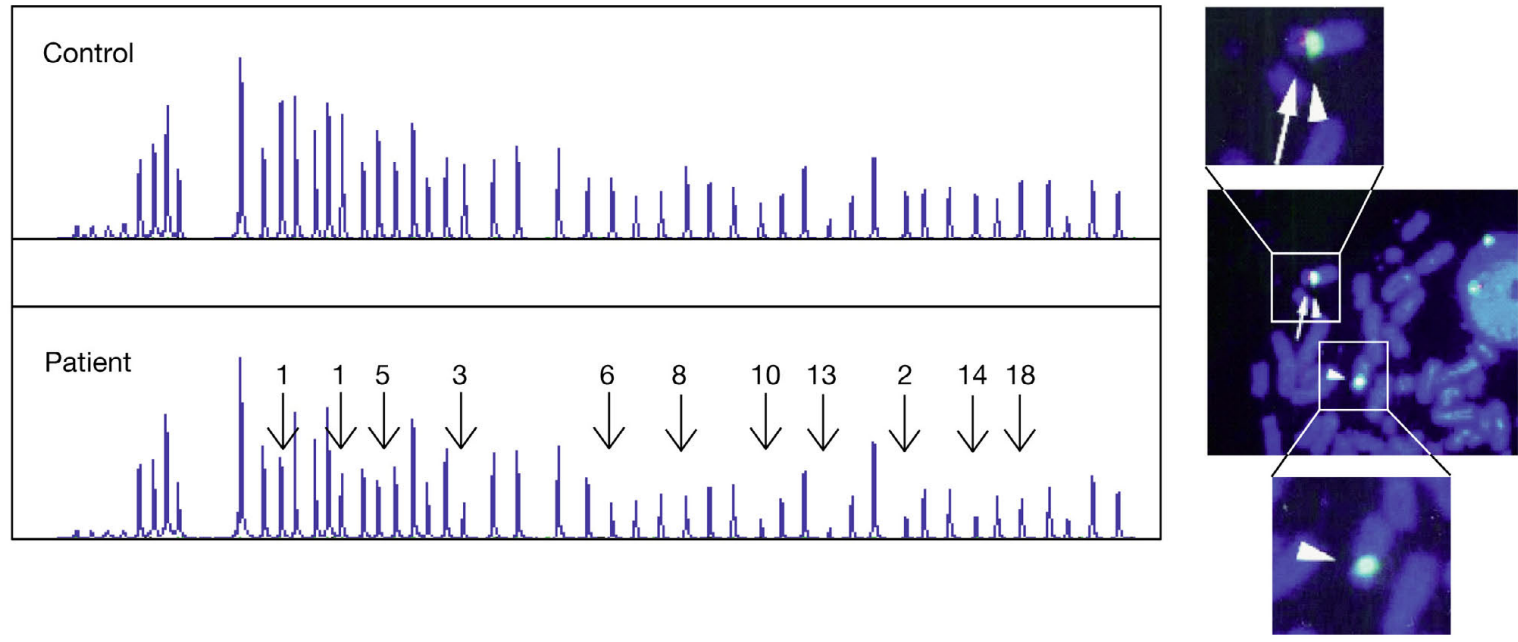

B


Fig. 1 Submicroscopic deletion involving FGFR1

A Deletion analyses. Left: MLPA analysis. The arrows indicate the peaks that correspond to FGFR1 exons. Reduced peak heights of MLPA probes suggest a heterozygous deletion. Right: FISH analyses on lymphocyte metaphase spreads. The FGFR1 probe detects only a single red signal (an arrow), whereas the chromosome 8 centromere probe (D8Z2) identifies two green signals (arrowheads). B Array-based CGH analysis. CGH indicated an $\sim 8.5 \mathrm{Mb}$ deletion at 8 p11.1-p12 involving FGFR1, together with a small duplication at 8p23.2 that is known as a benign polymorphism (Database of Genomic Variants; http://projects.tcag.ca/variation/).

\section{Functional analysis of missense variants}

In vitro functional assays were carried out for the p.V102I and p.S107L variants (Fig. 2B and C). The relative luciferase activity of $\mathrm{p} . \mathrm{S} 107 \mathrm{~L}$ did not significantly differ from that of WT ( $p=0.0552)$. Furthermore, although $p$.V102I showed a slightly lower activity compared to WT ( $p=0.0413)$, there was no significant difference in activity between WT and p.V102I plus WT $(1: 1)(p=0.0872)$.

\section{Clinical features of the deletion-positive patient}

The deletion-positive patient presented with primary amenorrhea at 20 years of age. She had no family history of pituitary dysfunction; her non-consanguineous parents were clinically normal.

Clinical examinations revealed delayed pubertal development (breast, Tanner stage 3; pubic hair, stage 2-3; axillar hair, stage 1), in addition to short stature (-2.7 SD) and learning disability. She had several episodes of convulsion from eight months of age and was 
Table 2 Genes and pseudogenes involved in the deletion

\begin{tabular}{|c|c|c|}
\hline Gene symbol (Refseq) & Accession number & Description \\
\hline UNC5D & NM_080872 & unc-5 homolog D (C. elegans) \\
\hline KCNU1 & NM_001031836 & potassium channel, subfamily $\mathrm{U}$, member 1 \\
\hline ZNF703 & NM_025069 & zinc finger protein 703 \\
\hline ERLIN2 & NM_007175 & ER lipid raft associated 2 \\
\hline LOC728024 & NR_003671 & chromosome X open reading frame 56 pseudogene \\
\hline PROSC & NM_007198 & proline synthetase co-transcribed homolog (bacterial) \\
\hline GPR124 & NM_032777 & G protein-coupled receptor 124 \\
\hline BRF2 & NM_018310 & subunit of RNA polymerase III transcription initiation factor, BRF1-like \\
\hline RAB11FIP1 & NM_025151 & RAB11 family interacting protein 1 (class I) \\
\hline GOT1L1 & NM_152413 & glutamic-oxaloacetic transaminase 1-like 1 \\
\hline ADRB3 & NM_000025 & adrenergic, beta-3-, receptor \\
\hline EIF4EBP1 & NM_004095 & eukaryotic translation initiation factor $4 \mathrm{E}$ binding protein 1 \\
\hline ASH2L & NM 004674 & ash2 (absent, small, or homeotic)-like (Drosophila) \\
\hline STAR & NM_000349 & steroidogenic acute regulatory protein \\
\hline LSM1 & NM_014462 & LSM1 homolog, U6 small nuclear RNA associated (S. cerevisiae) \\
\hline BAG4 & M_004874.3 & BCL2-associated athanogene 4 \\
\hline DDHD2 & NM_001164234 & DDHD domain containing 2 \\
\hline PPAPDC1B & NM_001102559 & phosphatidic acid phosphatase type 2 domain containing $1 \mathrm{~B}$ \\
\hline WHSC1L1 & NM_017778 & Wolf-Hirschhorn syndrome candidate 1-like 1 \\
\hline LETM2 & NM_144652 & leucine zipper-EF-hand containing transmembrane protein 2 \\
\hline FGFR1 & NM_023105 & fibroblast growth factor receptor 1 \\
\hline C8orf86 & NM_207412 & chromosome 8 open reading frame 86 \\
\hline RNF5P1 & NR_003129 & ring finger protein 5, E3 ubiquitin protein ligase pseudogene 1 \\
\hline TACC1 & NM_001122824 & transforming, acidic coiled-coil containing protein 1 \\
\hline PLEKHA2 & NM_021623 & pleckstrin homology domain containing, family A member 2 \\
\hline HTRA4 & NM_153692 & HtrA serine peptidase 4 \\
\hline TM2D2 & NM_001024381 & TM2 domain containing 2 \\
\hline ADAM9 & NR_027639 & ADAM metallopeptidase domain 9 \\
\hline ADAM32 & NM_145004 & ADAM metallopeptidase domain 32 \\
\hline ADAM5P & NR_001448 & ADAM metallopeptidase domain 5 , pesudogene \\
\hline ADAMЗА & NR_001569 & ADAM metallopeptidase domain 3A \\
\hline LOC100130964 & NR_046245 & ADAM metallopeptidase domain 3A-like \\
\hline ADAM18 & NM_014237 & ADAM metallopeptidase domain 18 \\
\hline ADAM2 & NM_001464 & ADAM metallopeptidase domain 2 \\
\hline IDO1 & NM_002164 & indoleamine 2,3-dioxygenase 1 \\
\hline IDO2 & NM_194294 & indoleamine 2,3-dioxygenase 2 \\
\hline C8orf4 & NM_020130 & chromosome 8 open reading frame 4 \\
\hline ZMAT4 & NM_001135731 & zinc finger, matrin-type 4 \\
\hline SFRP1 & NM_003012 & secreted frizzled-related protein 1 \\
\hline GOLGA7 & NM_001174124 & golgin A7 \\
\hline GINS4 & NM_032336 & GINS complex subunit 4 \\
\hline AGPAT6 & NM_178819 & 1-acylglycerol-3-phosphate O-acyltransferase 6 \\
\hline NKX6-3 & NM_152568 & NK6 homeobox 3 \\
\hline ANK1 & NM_000037 & ankyrin 1 , erythrocytic \\
\hline MIR486 & NR_030161 & microRNA 486 \\
\hline KAT6A & NM_001099412 & K(lysine) acetyltransferase 6A \\
\hline AP3M2 & NM_006803 & adaptor-related protein complex 3 , mu 2 subunit \\
\hline PLAT & NM_033011 & plasminogen activator, tissue \\
\hline IKBKB & NM_001556 & inhibitor of kappa light polypeptide gene enhancer in B-cells, kinase beta \\
\hline POLB & NM_002690 & polymerase (DNA directed), beta \\
\hline DKK4 & NM_014420 & dickkopf homolog 4 (Xenopus laevis) \\
\hline VDAC3 & NM_005662 & voltage-dependent anion channel 3 \\
\hline SLC20A2 & NM_001257180 & solute carrier family 20 (phosphate transporter), member 2 \\
\hline C8orf40 & NM_138436 & chromosome 8 open reading frame 40 \\
\hline CHRNB3 & NM_000749 & cholinergic receptor, nicotinic, beta 3 \\
\hline CHRNA6 & NM_001199279 & cholinergic receptor, nicotinic, alpha 6 \\
\hline
\end{tabular}


A

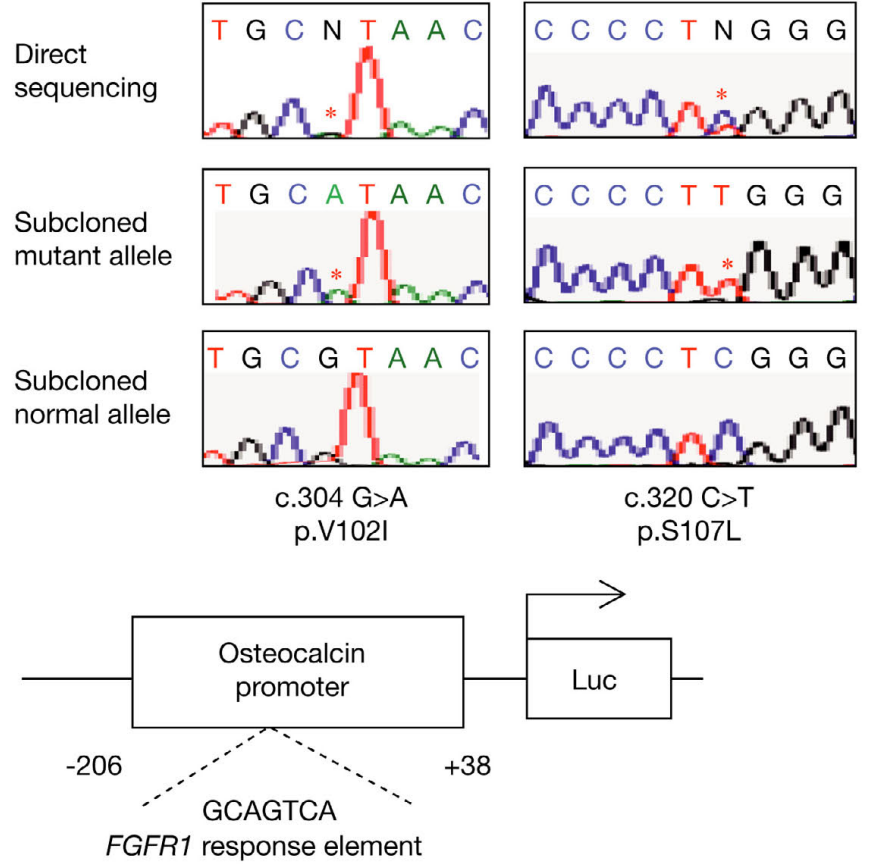

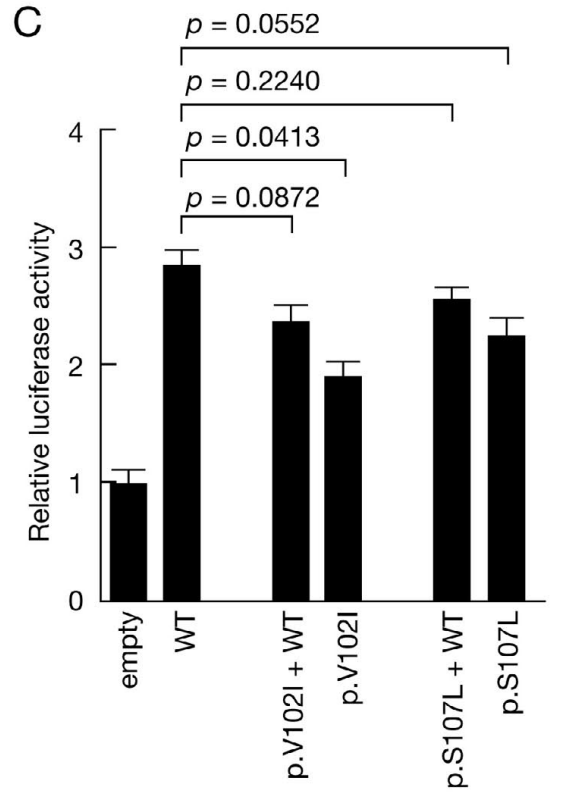

Fig. 2 Missense variations of FGFR1 identified in the present study

A Electrochromatograms delineating p.V102I and p.S107L variations. Asterisks indicate mutated nucleotides. B Luciferase reporter construct utilized in the present study. C Representative results of luciferase assays. Relative luciferase activities of the empty expression vector (empty), the wildtype vector (WT), the variant FGFR1 vectors (p.V102I and p. S107L), and WT plus the variant vectors $(1: 1)$ are shown.
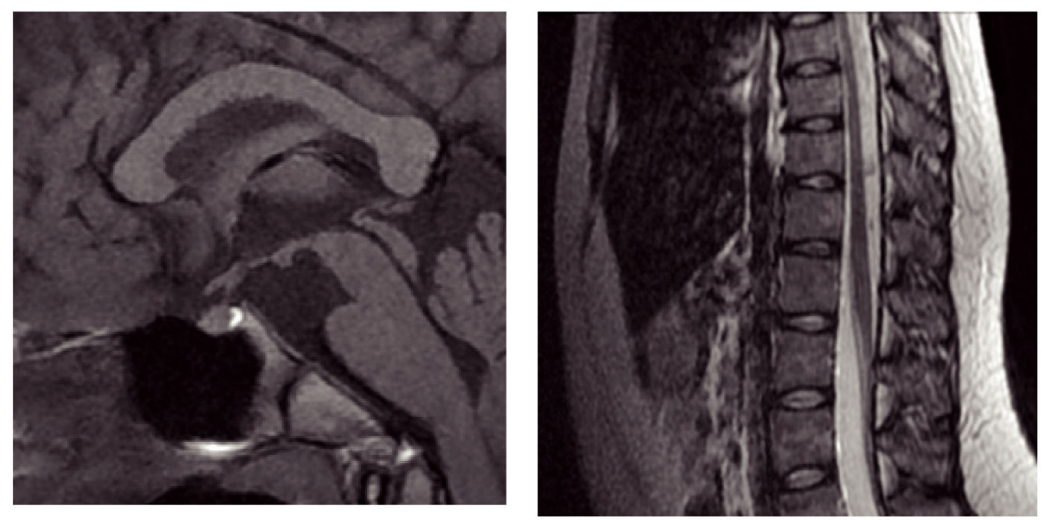

Fig. 3 Brain magnetic resonance imaging of the deletion-positive patient

Chiari type I malformation, syringomyelia, and apparently normal pituitary and stalk are shown.

diagnosed as having epilepsy. Brain magnetic resonance imaging delineated Chiari type I malformation and syringomyelia, together with an apparently normal pituitary and stalk (Fig. 3). She had a normal sense of smell, although we did not perform a quantitative examination of olfactory function.

Endocrine evaluation delineated a combined deficiency of pituitary hormones (Table 3). Blood GH lev- els were normal at the baseline, but did not increase after insulin stimulation. Gonadotropin levels were low at the baseline, and did not respond to GnRH stimulation. Basal and TRH-stimulated values of TSH remained within the reference ranges, while the basal level of free T4 was low. Basal and TRH-stimulated levels of prolactin were normal. 
Table 3 Endocrine data of the deletion-positive patient

\begin{tabular}{lcccc}
\hline Hormone & Stimulus (dosage) & Basal value & Peak value $^{\text {Peak response }}$ \\
\hline $\mathrm{GH}(\mathrm{ng} / \mathrm{mL})^{\mathrm{b}}$ & Insulin $(0.1 \mathrm{U} / \mathrm{kg})^{\mathrm{c}}$ & $4.3(0.2-23.0)$ & $\mathbf{4 . 4}(>6.0)$ & at $90 \mathrm{~min}$. \\
$\mathrm{LH}(\mathrm{mIU} / \mathrm{mL})$ & GnRH $\left(100 \mu \mathrm{g} / \mathrm{m}^{2}\right)$ & $\mathbf{0 . 1}(0.4-4.1)$ & $\mathbf{0 . 7}(8.5-15.5)$ & no peak \\
FSH $(\mathrm{mIU} / \mathrm{mL})$ & $\mathrm{GnRH}\left(100 \mu \mathrm{g} / \mathrm{m}^{2}\right)$ & $\mathbf{0 . 4}(4.8-10.4)$ & $\mathbf{1 . 6}(8.3-20.2)$ & no peak \\
$\mathrm{TSH}(\mu \mathrm{U} / \mathrm{mL})$ & $\mathrm{TRH}(10 \mu \mathrm{g} / \mathrm{kg})$ & $2.4(0.9-5.0)$ & $10.8(5.0-26.5)$ & at $30 \mathrm{~min}$. \\
Prolactin $(\mathrm{ng} / \mathrm{mL})$ & $\mathrm{TRH}(10 \mu \mathrm{g} / \mathrm{kg})$ & $7.2(1.4-14.6)$ & $18.9(>13)$ & at $30 \mathrm{~min}$. \\
ACTH $(\mathrm{pg} / \mathrm{mL})$ & Insulin $(0.1 \mathrm{U} / \mathrm{kg})^{\mathrm{c}}$ & $54.4(7.4-55.7)$ & $89.0(>30)$ & at $60 \mathrm{~min}$. \\
Cortisol $(\mu \mathrm{g} / \mathrm{dL})$ & Insulin $(0.1 \mathrm{U} / \mathrm{kg})^{\mathrm{c}}$ & $13.4(3.9-21.3)$ & $18.9(>18.0)$ & at $60 \mathrm{~min}$. \\
Estradiol $(\mathrm{pg} / \mathrm{mL})$ & & $\mathbf{1 4}(36-113)$ & & \\
Free T4 $(\mu \mathrm{g} / \mathrm{dL})$ & & $\mathbf{0 . 4}(0.9-1.7)$ & & \\
IGF1 $(\mathrm{ng} / \mathrm{mL})$ & & $200(119-389)$ & & \\
\hline
\end{tabular}

The conversion factor to the SI unit: GH, 1.0 ( $\mu \mathrm{g} / \mathrm{L})$; LH 1.0 (IU/L), FSH 1.0 (IU/L), TSH, 1.0 (mIU/L); ACTH, 0.22 (pmol/L); cortisol, 27.59 (nmol/L); estradiol, 3.671 (pmol/L); free T4, 12.87 (pmol/L) and IGF1, 0.131 (nmol/L).

Reference range in age-matched individuals are shown in parenthesis. Hormone values below the reference range are boldfaced.

${ }^{a}$ Blood sampling during the provocation tests: 0, 30, 60, 90, and 120 minutes. ${ }^{\mathrm{b}} \mathrm{GH}$ was measured using the recombinant $\mathrm{GH}$ standard, and the peak $\mathrm{GH}$ value of $6.0 \mathrm{ng} / \mathrm{mL}$ are used as the cutoff value for $\mathrm{GH}$ deficiency.

${ }^{\mathrm{c}}$ Sufficient hypoglycemic stimulations were obtained during all the insulin provocation tests.

\section{Discussion}

Copy-number analyses identified a submicroscopic deletion involving FGFR1 in one of 69 patients with CPHD. The phenotype of the deletion-positive patient, such as brain anomalies and CPHD, is likely be associated with FGFR1 haploinsufficiency, because none of 55 other genes involved in the deletion are known to play a role in the brain development (NCBI database, http://www.ncbi.nlm.nih.gov/; UCSC Genome Browser, http://genome.ucsc.edu/). However, we could not exclude the possibility that this deletion is a nonpathogenic variant, because parental samples of the patient were not available for genetic analysis. In this regard, it is worth mentioning that previous studies have shown that FGFR1 is clearly expressed throughout the neuroepithelium of the developing brain including Rathke's pouch [6], and FGF signaling regulates the proliferation and differentiation of cultured pituitary progenitor cells $[9,10]$. In addition, the CPHD phenotype has also been described in a patient with a large interstitial deletion on 8p11.2 affecting FGFR1 [8]. These results, in conjunction with previous findings that FGFR1 mutations and deletions are present in a certain part of patients with $\mathrm{IHH}, \mathrm{KS}$, and SOD $[3,7]$, suggest that FGFR1 abnormalities can be associated with various developmental defects of the anterior midline in the forebrain. Nevertheless, CPHD seems to be a rare manifestation among patients with
FGFR1 abnormalities, because the majority of previously reported patients with mutations or deletions of FGFR1 had no hormonal abnormalities with the exception of gonadotropin deficiency $[3,13]$. These results can be explained by assuming that during development, GnRH neurons are more vulnerable to FGFR1 abnormalities than the other parts of the anterior midline in the forebrain. Furthermore, the high frequency of gonadotropin deficiency and the rarity of other pituitary hormone deficiencies in patients with FGFR1 abnormalities are consistent with the results of in vitro assays; Norlin et al. have suggested that in the developing pituitary, FGFs play a particularly important role in the specification of the POU1F1-independent progenitor cells including gonadotrophs [9].

The deletion-positive patient manifested a combined deficiency of GH, LH and FSH. In addition, normal TSH values along with a low free T4 level are indicative of central hypothyroidism [14]. Since endocrine evaluation of this patient was fragmentary, it remains unknown whether the primary lesion of her hormonal defects resides in the hypothalamus or in the pituitary. Further studies, such as GHRH stimulation tests or GnRH stimulation tests after $\mathrm{GnRH}$ priming, will clarify the origin of CPHD in patients with FGFR1 abnormalities.

Mutation analysis identified two heterozygous FGFR1 variations (p.V102I and p.S107L) in two of 69 patients with CPHD. In vitro assays indicated that heterozygosity of the p.V102I and p.S107L vari- 
ants resulted in nearly normal transactivating function. Furthermore, p.V102I and p.S107L were found in a small subset of unaffected individuals. Thus, these two substitutions are more likely to be benign polymorphisms rather than disease-causing mutations. These results imply that FGFR1 intragenic mutations account for only a minor part of the etiology of CPHD, if any. However, given the relatively small number of patients analyzed in the present study, further studies are necessary to clarify the contribution of FGFR1 mutations to the development of CPHD.

In summary, the present study indicates a possible role of FGFR1 in anterior pituitary function and the rarity of FGFR1 abnormalities in patients with CPHD.

\section{Acknowledgements}

This work was supported by grants from the Ministry of Health, Labor and Welfare and from Takeda Science Foundation, by Grant-in-Aid for Scientific Research from the Japan Society for the Promotion of Science, by Grant-in-Aid for Scientific Research on Innovative Areas from the Ministry of Education, Culture, Sports, Science and Technology and by the Grant of National Center for Child Health and Development.

\section{Disclosure}

None of the authors have any potential conflicts of interest associated with this research.

\section{References}

1. Miraoui H, Dwyer A, Pitteloud N (2011) Role of fibroblast growth factor (FGF) signaling in the neuroendocrine control of human reproduction. Mol Cell Endocrinol 346: 37-43.

2. Mohammadi M, Olsen SK, Ibrahimi OA (2005) Structural basis for fibroblast growth factor receptor activation. Cytokine Growth Factor Rev 16: 107-137.

3. Villanueva C, de Roux N (2010) FGFR1 mutations in Kallmann syndrome. Front Horm Res 39: 51-61.

4. Falardeau J, Chung WC, Beenken A, Raivio T, Plummer L, et al. (2008) Decreased FGF8 signaling causes deficiency of gonadotropin-releasing hormone in humans and mice. J Clin Invest 118: 2822-2831.

5. Sykiotis GP, Plummer L, Hughes VA, Au M, Durrani S, et al. (2010) Oligogenic basis of isolated gonadotropinreleasing hormone deficiency. Proc Natl Acad Sci USA 107: 15140-15144.

6. McCabe MJ, Gaston-Massuet C, Tziaferi V, Gregory LC, Alatzoglou KS, et al. (2011) Novel FGF8 mutations associated with recessive holoprosencephaly, craniofacial defects, and hypothalamo-pituitary dysfunction. $J$ Clin Endocrinol Metab 96: E1709-1718.

7. Raivio T, Avbelj M, McCabe MJ, Romero CJ, Dwyer AA, et al (2012) Genetic Overlap in Kallmann Syndrome, Combined Pituitary Hormone Deficiency, and Septo-Optic Dysplasia. J Clin Endocrinol Metab 97: E694-699.

8. Vermeulen S, Messiaen L, Scheir P, De Bie S, Speleman F, et al. (2002) Kallmann syndrome in a patient with congenital spherocytosis and an interstitial 8p11.2 dele- tion. Am J Med Genet 108: 315-318.

9. Norlin S, Nordström U, Edlund T (2000) Fibroblast growth factor signaling is required for the proliferation and patterning of progenitor cells in the developing anterior pituitary. Mech Dev 96: 175-182.

10. Ericson J, Norlin S, Jessell TM, Edlund T (1998) Integrated FGF and BMP signaling controls the progression of progenitor cell differentiation and the emergence of pattern in the embryonic anterior pituitary. Development 125: 1005-1015.

11. Raivio T, Sidis Y, Plummer L, Chen H, Ma J, et al. (2009) Impaired fibroblast growth factor receptor 1 signaling as a cause of normosmic idiopathic hypogonadotropic hypogonadism. J Clin Endocrinol Metab 94: 4380-4390.

12. Newberry EP, Boudreaux JM, Towler DA(1996) The rat osteocalcin fibroblast growth factor (FGF)-responsive element: an okadaic acid-sensitive, FGF-selective transcriptional response motif. Mol Endocrinol 10: 10291040.

13. Tsukahara M, Murano I, Aoki Y, Kajii T, Furukawa S (1995) Interstitial deletion of 8p: report of two patients and review of the literature. Clin Genet 48: 41-45.

14. Salvatore D, Davies TF, Schlumberger M-J, Hay ID, Larsen PR (2011) Thyroid physiology and diagnostic evaluation of patients with thyroid disorders. In: Melmed S, Polonsky KS, Larson PR, Kronenberg HM (ed) Williams Textbook of Endocrinology (12th). Saunders, Philadelphia: 327-361. 